\title{
Research on Formation Method of Multi-robot
}

\author{
Qinzhao Wang ${ }^{1, \mathrm{a}}$, Jinyong Cheng ${ }^{1, \mathrm{~b},{ }^{*}}$, Xiaolong $\mathrm{Li}^{1, \mathrm{c}}$, Jin Pei ${ }^{2}$, Junxiao Bao ${ }^{1}$ \\ ${ }^{1}$ Department of Control Engineering, Armored Force Engineering College, Beijing100072, China; \\ ${ }^{2} 1$ st Brigade, Fourth Military Medical University, Xi'an 710032, China; \\ ackingcourage@163.com, bckingcourage@126.com, '13261558070@163.com
}

Keywords: multi-robot; formation; differential evolution algorithm; artificial potential field

\begin{abstract}
Aiming at the formation problem of multi-robot formation, a phased multi-robot formation strategy is proposed. The formation strategy takes full account of the robot's pose information and formation cost constraints, and decomposes the formation problem into two stages: the formation point distribution and the motion control. An improved discrete differential evolution algorithm is used to allocate the appropriate formation point for the robot. The motion controller is constructed using the consistency theory and the potential field idea, so that the robot at any initial position can move to the formation point without safety. Simulation results show that the formation strategy can form an effective formation, reduce the resource consumption of the robot during the formation process and improve the formation efficiency.
\end{abstract}

\section{Introduction}

Multi-robot formation control refers to a number of robots in the process of moving to the target both to maintain a certain geometric formation, but also to adapt to the constraints of the environment, safe obstacle avoidance. Multi-robot formation control technology in resource exploration, security patrol, earthquake relief and military operations and other aspects have a very broad application prospects. The formation of multiple robots is one of the fundamental problems of multi-robot formation. The literature [1] uses the relative distance to determine the formation and uses the distributed navigation function to form the formation. In [2], the desired formation is formed by the autonomous control rate. In [3], the optimal formation strategy is proposed to study the formation of static initial formation of multiple robots. In [4], the multi-robot formation control strategy based on bidding mechanism is used to study the rapidity of formation.

In this paper, a phased formation control strategy is used to solve the problem of formation of multi-robot formation. The robot and the formation point are allocated by the improved discrete difference evolution algorithm, integer Coding method to construct population to initialize individuals, random elimination and replacement method of repetitive sequence and neighborhood search strategy. Motion control stage, the leadership to follow the problem into the robot on the formation point of the tracking problem, the use of consistent thinking and potential barrier strategy to build the robot motion controller, making the robot under the action of the controller to the assigned distribution Point movement. Finally, the effectiveness of the proposed method and the effectiveness of the formation strategy are verified by simulation experiments.

\section{Problem description}

The formation of multiple robots in the formation process, the robot from the chaotic state of disorder to orderly formation of the state usually need to face two big problems: (1) How to assign the formation point? When the robots in a disorganized state gather to a location area where they need to collaborate, unreasonable assignment of the formation points increases the likelihood of robot detours, increasing the cost of formation and formation time. (2) How the robot moves to the formation point? When the robot moves toward the selected formation, it may encounter obstacles 
or other robots, so the appropriate motion controller is needed to guide and control the behavior of the robot so that the robot can safely reach the scheduled formation position.

As shown in Fig.1, assume that the robot R1, R2, R3 of the formation points were T1, T2, T3, because the R3 path is short, first move to T3, so as to block R1 to reach the ideal path T1, R1 Forced to avoid obstacles, increased the number of obstacle avoidance and path length. If R1 and R3 are symmetrical, the ideal path for R3 to T1 is short, first moves to T3, and does not prevent R1 from reaching the ideal path of T3. The latter robots complete the task of team time is shorter, the total path of all robots is shorter, reducing the resource consumption and formation time.

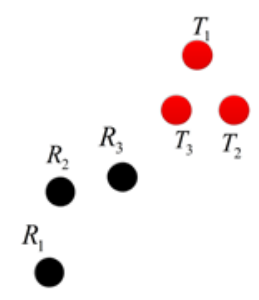

Fig. 1 Robot formation point allocation

\section{Formation point allocation stage}

Considering the problem that the distribution of the formation points is similar to the NP problem, we transform the problem into the task assignment problem, and uses the differential evolution algorithm (DE) to solve the problem. DE has the advantages of simple principle, less control parameters, different parameters of the parameters have little effect on the running result, with good global convergence and robustness. The basic idea is that the differential evolution algorithm is discretized, (Discrete Differential Evolution, DDE), which transforms and crossover the current population to produce another new population. Then, the selection of the two populations is selected by the choice of greedy ideas. The final generation of the next generation.

\subsection{Objective function}

The purpose of the assignment is to minimize the resource consumption of each robot at the formation point, while the total consumption of the overall consumption of the least. According to this, the multi-objective optimization function of robot formation assignment is established as shown in Equation (1) (2):

$$
\begin{gathered}
\min \left(\sum_{i=1}^{N} L_{i}\right) \\
\min \left(\max \left(L_{1}, L_{2}, L_{3}, \cdots, L_{N}\right)\right)
\end{gathered}
$$

$L_{i}$ represent the cost of the first robot $i$ to the formation point.

\subsection{Fitness function}

In this paper, two-wheel differential robot model analysis is used. Assuming that the robot is moving toward the formation point, it involves two operations in situ and in a straight line. The cost of the robot reaching the target point takes into account the distance between the robot and the target point and the turning angle of the robot. This section uses the "straight plus" way to convert the robot's turning angle to distance for processing. As shown in Fig.2, $\theta$ is the angle difference between the $\operatorname{robot}^{i}$ and the formation point ${ }^{j} \cdot \theta_{1} \theta_{2}$ is the angle between the robot and the formation point. Equation (3) transforms the angle difference into distance cost, $f(\Delta \theta)$ is the angle conversion function, ${ }^{l_{i j}}$ is the actual distance of the robot, $L_{i j}$ is the synthetic distance of the robot and the target point, respectively, $\nu, \omega$ are robot speed and angular velocity.

$$
\begin{aligned}
& \theta=\theta_{1}+\theta_{2} \\
& L_{i j}=a_{1} l_{i j}+a_{2} \Delta \theta \\
& a_{2}=\frac{v}{\omega}
\end{aligned}
$$


The fitness function is established by using the idea of penalty function, and the problem of multi - objective optimization of equation (1) and equation (2) is transformed into single objective optimization problem as shown in Equation (4).

$$
f=\lambda_{1} \sum_{i=1}^{N} L_{i}+\lambda_{2} \max \left(L_{1}, L_{2}, L_{3}, \cdots, L_{N}\right)
$$

\subsection{Algorithm solving}

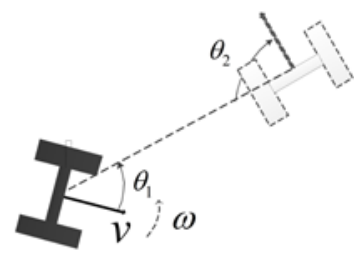

Fig. 1 Robot cost conversion model

(1) Coding: The integer distribution method is used to represent the distribution relation between the robot and the formation point as the initialization individual of the differential evolution algorithm. The complexity of the operator dimension and the operation is reduced by using the generated random integer sequence [5, 3, 2, 1, 4] instead of the traditional 0-1 matrix coding scheme. The sequence indicates that the assignments assigned by the robots R1, R2, R3, R4, R5 are T5, T3, T2, T1, T4, respectively.

(2) Mutation operator: Using the conventional DE / rand / 1 / bin mutation operator, the individual $x_{r_{0}, G}$ is mutated to produce mutated individuals $v_{i, G+1}$ as Equation (5).

$$
v_{i, G+1}=\operatorname{round}\left(x_{r_{0}, G}+F \cdot\left(x_{r_{1}, G}-x_{r_{2}, G}\right)\right)
$$

(3) Crossover operator: In Equation (6), mutation of the individual $v_{i, G+1}$ and the parent of the individual cross $x_{i j, G}$, resulting in individual $\mathrm{u}_{i j, G+1}$, and $\operatorname{rand}(j)$ is a random number, $C R$ is the crossover probability, $r n b r(i)$ is the random dimension of the problem $\operatorname{rand}(j)$ dimension.

$$
\mathrm{u}_{i, G+1}=\left\{\begin{array}{l}
v_{i, G+1}, \text { 若 } r a n d(j) \leq C R \text { 或 } j=r n b r(i) \\
x_{i j, G}, \text { 若 } \operatorname{rand}(j)>C R \text { 且 } j \neq \operatorname{rnbr}(i)
\end{array}(i=1,2, \cdots N P ; j=1,2, \cdots D)\right.
$$

In the process of crossing, there is a solution that the individual does not satisfy the constraint, and it is necessary to deal with the infeasible solution. As shown in Fig. 3, a non-repetitive sequence in $\mathrm{u}_{i j, G+1}$ individual is retained, and for repetitive sequences, the repeated values are retained in a randomly selected manner. For the missing sequence of numbers of $\mathrm{u}_{i, G+1}$, the corresponding operator in parent $x_{i j, G}$ is randomly inserted into the vacant position, and the new sequence is generated as the test entity $\mathrm{u}_{i, G+1}$.
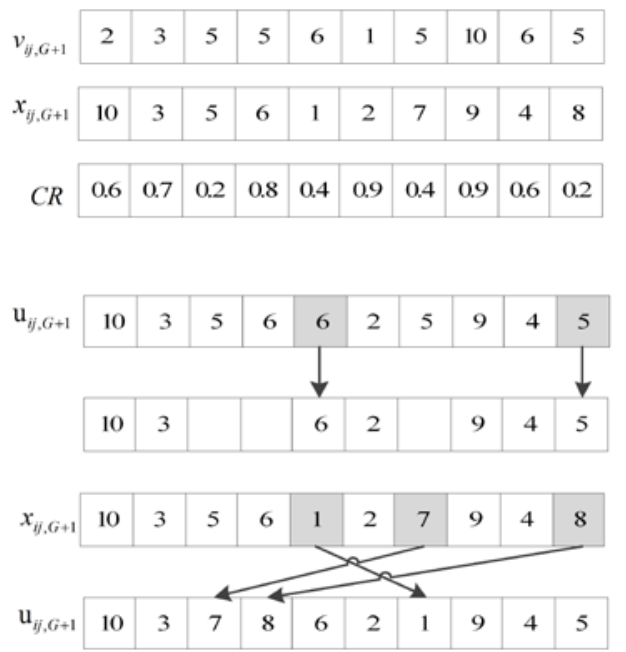

Fig. 3 Repeat the encoding operation 
(4) Select the operation

By using the "greed" strategy, we compared the fitness function of the offspring and the father, and choose the next generation of individual population $x_{i}^{t+1}$ as Equation (7).

$$
x_{i}^{t+1}=\left\{\begin{array}{l}
u_{i}^{t+1}, f\left(u_{i}^{t+1}\right)<f\left(x_{i}^{t}\right) \\
x_{i}^{t}, \text { 其他 }
\end{array}\right.
$$

\section{Motion control phase}

Aiming at the problem of robot movement planning, common methods are raster method, visual graph method, group intelligent algorithm, artificial potential field method, etc., which is a more mature artificial field method, the method is to move the robot movement The space is regarded as the abstract potential space, and the path planning is carried out through the potential field model. The target points apply gravitational force to the robot, and the obstacle applies repulsive force to the robot. The two forces form a joint force to control the movement state of the robot. The structure of the potential field is relatively simple, the mathematical calculation is simple and clear, which is convenient for real-time control and the planning path is smooth and safe.

Based on the theory of artificial potential and the basic theory of consistency proposed by Reynolds in [5], the robot motion controller is designed to prevent the robot from colliding with obstacles or other robots while moving toward a predetermined formation point. This paper designs the robot controller for:

$$
u=F_{i}^{1}+F_{i}^{2}+F_{i}^{3}
$$

Among them, $F_{i}^{1}$ for the $i$ robot by the team point of attraction, $F_{i}^{2}$ for the collision, $F_{i}^{3}$ for the barrier.

\subsection{Attraction of the formation point}

The formation point of the formation point for the gravitational potential field, the robot in the formation of gravity under the action of gravity, gravitational potential and gravity respectively is Equation (9) 、Equation (10).

$$
\begin{gathered}
\psi_{1}=\frac{1}{2} k_{1}\left|x_{\mathrm{r}}-x_{i}\right|^{2} \\
F_{i}^{1}=\nabla \psi_{1}=-k_{1}\left(x_{i}-x_{r}\right)
\end{gathered}
$$

Where, $\psi_{1}$ is the gravitational field function, $F_{i}^{1}$ is the gravitational force of the robot, $k_{1}$ is the gain coefficient, $x_{i}, x_{r}$ are the position of the robot position and the formation point respectively.

\subsection{Collision Avoidance Control}

When the robot moves toward the formation point, the movement path of the robot may cross, and the robot may collide. In order to realize the collision avoidance between robots, the collision potential function is a differentiable, nonnegative and unbounded function on the distance of the robot. The distance between the two robots is $d$, the actual relative The distance is $x, x$ is less than $d$ for the repulsion, so that the distance between the robot increases.

The collision potential function and the avoidance force are defined as Equation (11) and Equation (12):

$$
\begin{gathered}
\psi_{2}= \begin{cases}0.5 \cdot k_{2} \cdot(1 / x-1 / d) & 0 \leq x \leq d \\
0 & x \geq d\end{cases} \\
F_{1}^{2}=\nabla \psi_{2}=-0.5 \cdot k_{2} \cdot\left(1 / x^{2}\right)
\end{gathered}
$$

Where $\psi_{2}$ is the potential field function, $k_{2}$ is the gain coefficient, $d$ is the influence range of the potential field, $F_{i}^{2}$ is the avoidance force.

\subsection{Obstacle avoidance control}

The obstacle avoidance is needed to detect the obstacle during the movement of the robot. The obstacle avoidance function is in the same form as the collision avoidance function. The gain coefficient is changed to $k_{3}$, and the repulsive influence range is $R$. 


\section{Simulation experiment}

The simulation experiments need to verify the two stages of the method respectively. Therefore, two simulation experiments are designed. First, the performance of the algorithm is verified by simulation experiment 1 . Secondly, the whole formation process is verified by simulation experiment 2. $N P=60, N G=400, \lambda_{2}=1, a_{1}=1, a_{2}=1 / 4, k_{1}=3, k_{2}=60, k_{3}=60, d=3, R=10$.

\subsection{Experiment 1}

Experiments were carried out using 15 formation points and 15 randomly distributed robots for assignment of formation points. The positions of the robot and the target are shown in Table 1 and Figure 2. (GA), the standard differential evolution algorithm (DE), and the genetic algorithm (GA) used in the literature [6] and the particle swarm of the literature [7] are used in this paper. Algorithm (PSO) were tested 20 times, the experimental results shown in Table 3.

As can be seen from Table 3, due to the complex cross and mutation operation, resulting in GA algorithm running speed is relatively slow, and easy to fall into the local optimal solution. PSO and DE run faster, but it is difficult to converge to the optimal solution, poor search ability. Experiments show that GDE combines the convergence speed and optimization ability of the original differential evolution method, and can quickly find a reasonable result of the distribution of the formation points, and the assignment of the formation points is effective. The optimal assignment results are: T8, T6, T4, T15, T11, T13, T2, T1, T9, T12, T7, T10, T14, T3, T5.

Table 1 Robot pose distribution

\begin{tabular}{cccccccccccc}
\hline number & $\mathrm{X}$ & $\mathrm{Y}$ & angle & number & $\mathrm{X}$ & $\mathrm{Y}$ & angle & number & $\mathrm{X}$ & $\mathrm{Y}$ & angle \\
\hline 1 & 20 & 20 & 60 & 6 & 75 & 80 & -145 & 11 & 20 & 30 & 0 \\
\hline 2 & 50 & 15 & 60 & 7 & 52 & 90 & -90 & 12 & 50 & 35 & -80 \\
\hline 3 & 83 & 39 & 135 & 8 & 49 & 88 & -90 & 13 & 80 & 49 & 90 \\
\hline 4 & 75 & 43 & 0 & 9 & 10 & 90 & -45 & 14 & 80 & 55 & 150 \\
\hline 5 & 85 & 95 & -135 & 10 & 12 & 50 & 45 & 15 & 85 & 15 & 145 \\
\hline
\end{tabular}

Table 2 Position of the formation point

\begin{tabular}{cccccccccccc}
\hline number & $\mathrm{X}$ & $\mathrm{Y}$ & angle & number & $\mathrm{X}$ & $\mathrm{Y}$ & angle & number & $\mathrm{X}$ & $\mathrm{Y}$ & angle \\
\hline 1 & 42 & 62 & 0 & 6 & 58 & 36 & 0 & 11 & 52 & 62 & 0 \\
\hline 2 & 50.5 & 65 & 0 & 7 & 42 & 37 & 0 & 12 & 20.5 & 65 & 0 \\
\hline 3 & 59 & 61 & 0 & 8 & 38 & 44 & 0 & 13 & 49 & 61 & 0 \\
\hline 4 & 63 & 51 & 0 & 9 & 38 & 55 & 0 & 14 & 73 & 51 & 0 \\
\hline 5 & 62.5 & 44 & 0 & 10 & 50 & 34 & 0 & 15 & 82.5 & 44 & 0 \\
\hline
\end{tabular}

Table 3 Comparison of GDE, DE, GA, PSO algorithms

\begin{tabular}{ccccccc}
\hline algorithm & $\begin{array}{c}\text { average } \\
\text { fitness }\end{array}$ & $\begin{array}{c}\text { optimal } \\
\text { fitness }\end{array}$ & $\begin{array}{c}\text { worst } \\
\text { fitness }\end{array}$ & $\begin{array}{c}\text { fitness } \\
\text { variance }\end{array}$ & $\begin{array}{c}\text { optimal } \\
\text { percentage }\end{array}$ & $\begin{array}{c}\text { run } \\
\text { time }\end{array}$ \\
\hline GA & 466.0834 & 460.9632 & 495.0328 & 112.1377 & 75 & $5.810 \mathrm{~s}$ \\
\hline DE & 491.2613 & 460.9632 & 526.2254 & 286.042 & 10 & $3.256 \mathrm{~s}$ \\
\hline PSO & 506.7056 & 477.8679 & 539.3061 & 323.6138 & 0 & $3.612 \mathrm{~s}$ \\
\hline GDE & 463.0414 & 460.9632 & 481.7463 & 38.8744 & 90 & $4.156 \mathrm{~s}$ \\
\hline
\end{tabular}

\subsection{Experiment 2}

The simulation environment for the two-dimensional region, there are obstacles in the area. Using the top 10 robots in Table 1 and the correspondent 10 target points in Table 2, the formation experiment was carried out. Assuming that all robots are working properly, the robot is represented by a red dot, with a short arrow, and the formation is indicated by an asterisk. The formation process is shown in Fig.4. The simulation results show that the proposed method is effective and can be used to realize the formation of the robot formation. The simulation results show that the formation of the robot is effective in the formation of the robot formation. The R9 and R1 robots encounter obstacles, the robot R5, R6 path cross, but the R6 run faster to reach the intersection, will not have an impact on R5. 


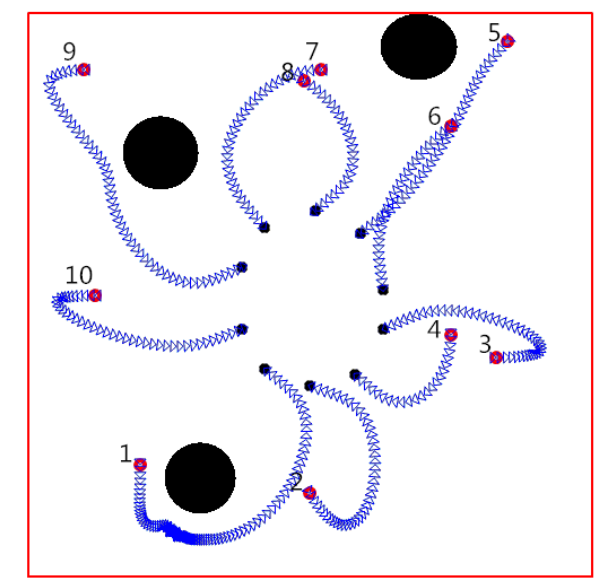

Fig. 4 multi-robot composition concentric formation

\section{Conclusion}

In this paper, the formation process of multi-mobile robot formation is decomposed into two stages: assignment of maneuver point and robot motion control, which simplifies the problem solving process. By using a large number of experiments, it is found that the hybrid differential evolution algorithm allows the robots to take into account the computing time and the optimization ability, select the optimal formation point, reduce the detour and collision, and quickly and smoothly make the arbitrary team shape. The movement of the robot to the movement of the controller can effectively avoid obstacles and avoid collision, the successful movement to the formation point. Compared with the previous method, the control scheme proposed in this paper is more convenient for the formation of the formation than the more complicated robot and the target formation.

\section{References}

[1] SUGIHARA K, SUZUKI I. Distributed algorithms for formation of geometric patterns with many mobile robots [J]. Journal of Robotic Systems, 1996, 13 (3): 127-139.

[2] FIERRO R, DAS A K, VIJIAY KUMARR, et al. Hybrid control of formations of robots [C] // Proceedings of the 2011 IEEE International Conference on Robotics and Automation Vol IEEE, 2001: 157-162.

[3] Dong Shenglong, Chen Weidong, Xi Yugeng. Time Optimal Formation of Mobile Robots [J]. Control and Decision, 2001,16 (5): 573-576.

[4] Yang Fan, Liu Shirong. Multi-robot real-time formation control [J]. Science and Technology Bulletin, 2010,26 (2): 205-212.

[5] Reynolds. Flocks, Birds, and Schools: A Distributed Behavior Model [J]. Computer Graphics, 1987, 21 (4): 25-34.

[6] DAI Gui-ping, WANG Yong, HOU Ya-rong. Algorithm for solving TSP problem based on genetic algorithm and its system [J]. Microcomputer Information, 2010 (04): 15-16,19.

[7] Yu Lei, Shi Feng, Wang Hui, etc. MATLAB intelligent algorithm [M]. 